\title{
PHYSICAL EXERCISE, STRESS, COPING, BURNOUT AND FATIGUE IN PERSONS WITH INCOMPLETE SPINAL CORD INJURY.
}

\author{
Anne M. Lannem \\ Department of Coaching and Psychology, Norwegian School of Sport Sciences and Sunnaas \\ Rehabilitation Hospital, Norway
}

The aim of this study was first to examine the prevalence of burnout and fatigue in a sample of persons with incomplete Spinal Cord Injury (SCI). The second aim was to investigate how selfperceptions of exercise and coping with disability-stress influenced the association between stress caused by disability and burnout or fatigue. A mailed survey was performed. A total of 186 persons, 142 males and 44 females, ages between $18-87$ years, were included in the study. Years post-injury ranged from 2 to 55 . The main findings were that $15 \%$ experienced burnout and $63 \%$ perceived fatigue or borderline fatigue. In order to perform path analysis, the method described by Baron and Kenny (1986) was used. Stress caused by the disability was indirectly linked to burnout and fatigue. Physical exercise and degree of coping with the disability-stress buffered the negative effects from stress caused by the disability on both burnout and fatigue.

Keywords: incomplete spinal cord injury, physical exercise, psychological aspects

\section{INTRODUCTION}

The level of physical activity is reported to be generally low in the population with spinal cord injury (SCI) (Buchholz, McGillivray, \& Pencharz, 2003). Consistent with the general population, physical exercise is recommended to prevent lifestyle diseases and enhance the quality of life and well-being for persons with SCI (Harvey, Lin, Glinsky, \& De, 2009; Hicks et al., 2003; Martin Ginis et al., 2003). However, a person with an incomplete SCI may be confronted with activity limitation due to their physical impairment in many daily life situations and also when exercising, and there is still limited knowledge regarding benefits from physical exercise particularly in persons with incomplete SCI. In addition, the literature is mostly on physiological and general psychological effects, and less is known about psychological processes and mechanisms related to physical exercise and persons with incomplete SCI. There is a need for a theoretical framework to inform these processes. In this study, the cognitive activation theory of stress (CATS) was used as a theoretical framework (Ursin \& Eriksen, 2004). The physical consequences of the SCI may be explained as stressors according to CATS. According to the theory, a homeostatic imbalance between the expected outcome and the factual outcome of a situation activates a neurophysiological stress alarm. The total health consequences of the stress alarm are determined by the way one reacts to the activation of the stress alarm. According to CATS, coping is defined as "positive outcome expectancy" (Ursin \& Eriksen, 2004). If the individual expects to be able to handle the stressful situation, i.e. has learned to cope effectively, the stress response or arousal level is reduced, and there are no further negative health consequences. If the imbalance persists, negative health effects such as burnout, fatigue or illness may occur. The consequences of an incomplete SCI may act as a persistent stressor. If, for example, a person with spasticity and limited walking abilities is always afraid of stumbling or falling, all obstacles, such as curbstones, uneven or slippery ground, a street with fast following traffic etc., can be perceived as stressors. The person's own low expectations to manage the situation may result in stumbling or falling. There are different possibilities to overcome such a stressor. Acceptance of reduced walking abilities is necessary for finding the best individual coping solution for outdoor mobility. One possible strategy is to increase strength, balance and walking ability with exercise and thereby prepare the person for outdoor ambulation. Another option is use of adaptive aids such as wheelchair or walking aids. Even if a person is able to cope with some of the stressors during the day, all together they may prove to be too much to handle. Coping with the stressors will be influenced by 
previous experiences and learning (Eriksen, Murison, Pensgaard, \& Ursin, 2005). The total rehabilitation process post-injury aims at preparing the persons with SCI to cope with such obstacles in everyday life by building protective levels of resources within physical, psychological and social fields. Acceptance of the consequences of the injury and realistic goal-setting is crucial for coping with the stressors due to the SCI (Kennedy, Evans, Berry, \& Mullin 2003; Roberts \& Kristiansen, 2012).

Realistic goal-setting should also include adjustment of expectations of recovery from being too high. This is a double edged sword as discussed by Lannem and Sørensen (in review). On the one hand, high expectations may enhance positive motivation to reach demanding goals. On the other hand, too high expectations may lead to defeat and increase the stress. Both too high and too low expectations from others, i.e. health professionals, may lead to frustration. Finally, while low expectation may simply reduce the stress, it may be that the person will not exploit his/her potential opportunities for maximum function.

In general, the rehabilitation process will influence both how stress is perceived in different situations, and how to cope with it. Lannem and Sørensen (in review) discussed how persons living at home with an incomplete SCI have to make active adjustments in order to reduce the physical demands in everyday life and thereby be able to cope better with the consequences of their activity limitations. As long as the level of physical, psychological and social resources are high enough and, the person believes that he/she can cope with the stressors, the resources may counteract the negative stress effects. If the physical demands in everyday life, however, are more than the person's total physical capacity, these resources may be overloaded. In the long run, they are likely to be reduced. Physical exercise in such a situation may be difficult to perceive as positive and instead turn into an extra stressor (Lannem \& Sorensen, in review). A long term outcome may result in overtraining and burnout, similar to what may happen to athletes in a sport context (Lemyre, Hall \& Roberts, 2008). Compared to an able-bodied person, it will be even more difficult to predict how much physical demands a person with SCI can tolerate before overtraining or burnout occur as a result of muscle weakness, lack of muscle control, weakened sensory input and autonomic reflexes resulting in reduced physical capacity.

The term and phenomenon burnout comes originally from studies on work-related stress. Work-related burnout is characterised as a psychological syndrome, including emotional exhaustion, depersonalisation, and reduced personal accomplishment (Maslach, 1993). Further, the reduced personal sense of accomplishment refers to: "a decline in one's feelings of competence and successful achievement in work" (p 21, Maslach, 1993). Based on this concept, Raedeke (1997) and Raedeke and Smith (2001) described athlete burnout as a multidimensional syndrome involving emotional and physical exhaustion, reduced sense of accomplishment in sport and devaluation of participation in sport. Lannem and colleagues argued that persons living with incomplete SCI may experience overload and burnout in relation to exercise (Lannem et al., 2010; Lannem \& Sorensen, in review). In addition, non-acceptance of technical aids, even if they need it, may increase overload of physical resources.

The role of physical exercise for the perception of stress and coping with stress was explored in persons with incomplete SCI in a qualitative study (Lannem \& Sorensen, in review). The main finding was that the total physical and psychological demands of everyday life influenced how exercise mastery was perceived. High exercise mastery was experienced when the exercise was perceived as building or maintaining physical resources. Low exercise mastery was experienced when the physical improvement stagnated, and when overload or fatigue developed.

The term fatigue can be defined as an overwhelming sense of tiredness, lack of energy and, often, a feeling of total exhaustion (Herlofson \& Larsen, 2002). While burnout has a stronger psychological background, prolonged fatigue is more related to a physical problem (Leone et al., 2010). In this study, the term fatigue is used as a symptom, and not as the diagnosis of Chronic Fatigue Syndrome (CFS) (Wessely, 2001). Fatigue is a frequently reported symptom in a large number of neurological disorders and is characterized by a feeling of constant exhaustion, including both a sense of physical exhaustion and an important cognitive component, a sense of mental fatigue (Chaudhuri \& Behan, 2004). Fatigue is also reported as an extra symptom for persons living 
with SCI (Anton, Miller \& Townson, 2008; Fawkes-Kirby et al., 2008).

According to CATS stress factors may accumulate and lead to burnout, fatigue, and chronic illness because of the physical impairments of persons with incomplete SCI, unless the challenges are coped with. Coping strategies may be physical exercise or the use of adaptive aids as mentioned above. Therefore, the purpose of the present study was: First, to determine the prevalence of burnout and fatigue in a Norwegian population of persons with incomplete SCI; and second, we wanted to analyse the role of physical exercise in the association between stress caused by the disability and burnout or fatigue. $\mathrm{We}$ hypothesized that the stress caused by the disability was indirectly linked to both burnout and fatigue, and that physical exercise and degree of coping with the perceived stress would buffer its negative effects.

\section{METHOD}

The design of the study was cross-sectional. Data was collected by a mailed questionnaire that measured perceived stress of the disability, leisure time physical activity, exercise mastery, coping, burnout and fatigue. Background information regarding injury level and severity, as well as additional injuries and complications, was collected by reviewing medical records.The AIS (ASIA impairment Scale) score $\mathrm{D}$ was used as the main inclusion criterion, and the injury level was divided into tetraplegia and paraplegia (Marino et al., 2003). The study was approved by the Regional Medical Research Ethics Committee, Eastern Norway. Respondents gave their written consent to participate. Two letters were sent prompting replies.

\section{Participants}

Included in the study were persons rehabilitated at Sunnaas Rehabilitation Hospital, Nesodden, Norway, between 1962 and 2008. Invitations were sent to 316 persons classified as AIS D or with a cauda equina injury. In order to minimize bias from general age changes, persons above 60 years at the time of the injury were excluded. Sample and injury characteristics are listed in Table 1.

\section{Measures}

Disability-related stress Furlong and Connor (2007) constructed a scale to measure disability-related stress in persons with physical disability. The Physical Disability Stress Scale (PDSS) was originally validated for adults with an acquired physical disability and who required the use of a wheelchair as their main source of mobility (Furlong \& Connor, 2007). The scale was translated into Norwegian using a double Translation - Back Translation technique. The total scale ( 22 items) may be used with 4 subscales, namely Access (4 items), Physical (5 items), Social (7 items) and Burden of care (6 items), or as a total scale score. The range of scores for the total scale is $0-110,0$ indicating not at all stressed/upset, and 110 indicating highly stressed/upset.

Exercise: Physical activity and physical exercise were measured by a 4-item rating of leisure time physical activity (LTPA) and the International Physical Activity Questionnaire (IPAQ).

LTPA: A one-item scale, developed in the 1960s and widely used in Norwegian health surveys, was used to measure leisure time physical activity (LTPA) (Graff-Iversen et al., 2008; Saltin \& Grimby, 1968). The respondents were asked to rate their weekly average LTPA: 1) Reading, watching TV or other sedentary activities; 2) Walking, cycling, using a wheelchair or moving about in some other way at least 4 hours per week - including commuting to work and Sunday walks; 3) Taking part in physical exercise or sports, heavy gardening work etc. - at least 4 hours a week; and 4) Exercising hard or taking part in competitive sports regularly several days a week. Wilhelmsen et al. (1968), Aires et al. (2003) and Graff-Iversen et al. (2008) have reported stable construct validity.

IPAQ: The IPAQ short version was developed for use with adults (18-69 years) (Craig et al., 2003). It collects information from the last 7 days, on time spent walking, doing moderate and vigorous-intensity activities and, also, time spent sitting. The instrument has shown acceptable reliability and validity (Craig et al., 2003). A Norwegian translation was available and had been used both for persons with and without a physical impairment (Saebu \& Sorensen, 2010; Wilhelmsen, 2009).

Perceived exercise mastery (PEM): In the mailed questionnaire PEM was defined as "a perception of competence when performing physical exercise" (Sorensen, 1997). The 
respondents were asked to rate their PEM at present on a visual analogue scale (VAS) ranging from $0-10(0=\mathrm{I}$ perceive no competence when performing physical exercise, $10=$ I perceive optimal competence when performing physical exercise). Aitken (1969) demonstrated that a $100 \mathrm{~mm}$ VAS captures slight differences in personal perceptions better than a categorical scale.

Degree of Coping: Coping was defined according to CATS as "positive outcome expectancy" (Ursin \& Eriksen, 2004). The respondents were asked to rate how they coped with the total physical demands in everyday life as experienced due to the SCI, on a $100 \mathrm{~mm}$ VAS rating from $0-10(0=$ no coping, $10=$ optimal coping).

Pines Burnout Measure (PBM): The 21 items in the PBM are scored on a 7-point Likert scale ranging from $1=$ never to $7=$ =always (Schaufeli et al., 1993). The scores can be recoded into a total burnout score (PBS). Average PBS which, when higher than 4, indicate burnout to the extent that mandatory changes in everyday life are necessary (Pines \& Aronson, 1988). The instrument has been tested and is considered a reliable and valid research instrument (Pines \& Aronson, 1988; Schaufeli, Enzmann, \& Girault, 1993). The PBM has been used as a measure for context-free burnout, and a Swedish national survey was used as comparable normative data (Hallsten, Bellagh, \& Gustafsson, 2002). The scale was translated to Norwegian using a double Translation Back Translation technique.

Fatigue Severity Scale (FSS): To measure fatigue, the FSS was used (Krupp, LaRocca, Muir-Nash, \& Steinberg, 1989). It consists of nine items, each scored on a Likert scale ranging from $1=$ "strong disagreement" to $7=$ "strong agreement". Reliability and validity of the FSS has been reported to be good in populations of persons with different impairments and normal healthy adults (Anton et al., 2008; Fawkes-Kirby et al., 2008; Krupp et al., 1989; Lerdal, Rustoen, Hanestad, \& Moum, 2005; Schanke \& Stanghelle, 2001). The cut-off score of over 4 , as indicative of fatigue, was originally set by Krupp et.al. (1989). In a Norwegian translation of the instrument and test of the psychometric properties, the investigators suggested that the threshold for defining severe fatigue should be a FSS score of 5 as opposed to the original score of 4 (Lerdal et al., 2005). Furthermore, they suggested that scores between 4 and 5 indicate borderline fatigue.

Statistical analyses: All analyses were conducted using PASW statistics 18.0 for Windows (IBM, SPSS statistics, Norway). Descriptive statistics were used to characterise the sample. Data was summarised by mean values and standard deviations (SD) or median values and range, when appropriate. Independent sample t-tests were used to compare mean values of dependent and independent variables in tetraplegia versus paraplegia. Due to the skewed distribution of the IPAQ data, the Spearman correlation test was used to measure the correlation between LTPA and the IPAQ. In order to test the mediations in Figure 1, the following three mediations were tested: Model 1) LTPA as a mediator in the path between PDSS and PEM; Model 2) Coping as a mediator in the path between PEM and PBS; Model 3) Coping as a mediator between PEM and FSS. In each model, linear regression equations were estimated as follows: First, regression of the mediator (LTPA in model 1, and Coping in model 2 and 3) on the independent variable (PDSS in model 1, and PEM in model 2 and 3); second, regression of the dependent variable (PEM in model 1, PBS in model 2 and FSS in model 3) on the independent variable (PDSS in model 1; and PEM in model 2 and 3); third, regression of the dependent variable (PEM in model 1, PBS in model 2 and FSS in model 3) on both the independent variable (PDSS in model 1, and PEM in model 2 and 3) and the mediator (LTPA in model 1 , and Coping in model 2 and 3) (Baron \& Kenny, 1986). Separate coefficients for each equation were estimated and tested using standard linear regression procedure. Pearson's correlation coefficient was used for correlations between variables in the mediation analyses.

\section{RESULTS}

\section{Participants}

A total of 316 persons fulfilled the inclusion criteria and received a mailed questionnaire. After two reminders, 199 persons (63\%) responded, either by returning the questionnaires or by a telephone interview. Seven persons refused to participate, and 4 persons were not able to answer due to presenile dementia. In addition, 2 persons turned 
out to be classified as AIS C and were consequently excluded from the analysis. The final sample consisted of 186 respondents. Because of ethical regulations in Norway, emphasized by the Regional Medical and Health Research Ethics, Eastern Norway and the Privacy Protection Supervisors, Oslo University Hospital, it was not possible to compare the respondents with the non- respondents without a written consent from the non-responders. The characteristics of the sample are given in Table 1. No differences were found between the subgroups tetraplegia versus paraplegia, for any of the dependent or independent variables (age, age at injury, duration of injury, sex, and additional illness/injury, PDSS, LTPA, PEM, Coping, PBS and FSS).

\section{TABLE 1}

Sample and injury characteristics $(n=186)$

\begin{tabular}{lcc}
\hline \hline & Mean (s.d.) & Range \\
\hline Age (years) & $48(14)$ & $18-87$ \\
Age at injury (years) & $33(14)$ & $5-60$ \\
Duration of injury (years) & $15(13)$ & $2-55$ \\
\hline & $\mathrm{n}$ & $\%$ \\
\hline Sex & & \\
Male & 142 & 76 \\
Female & 44 & 24 \\
Injury level & & \\
Tetraplegia & 85 & 46 \\
Paraplegia & 101 & 54 \\
Additional illness/injury & 80 & 43 \\
\hline
\end{tabular}

PDSS The population in this study included both persons using wheelchair and those who were walking compared with the original population which included only persons using wheelchair for mobility. As a result, the population in this study was more heterogeneous than the original population, and therefore we tested the internal consistency of the total scale in the studied population. The Chronbach Alfa coefficient was 0.93. On this background we used the total score. The mean total PDSS was $38.6(\mathrm{SD}=23.1)$, see Table 2 .

\section{Comparison of LTPA and the IPAQ}

In order to compare the measures LTPA and IPAQ in the study population, a correlation test was performed between LTPA (median 2, range 3) and IPAQ short version (median 3356 , range 22152). The correlation was reasonably high $(\mathrm{rs}=.55, \mathrm{p}<.001)$. However, all the IPAQ measures were highly skewed towards zero and LTPA had a normal distribution. It seems as though, in a less active population such as persons with incomplete SCI, LTPA captures more of the variance in the lower part of the scale, and was therefore used in the final analyses.

\section{Burnout and fatigue}

PBM Of the respondents, 173 persons (93\%) completed the PBM. The mean PBS was $2.8(\mathrm{SD}=1.2)$, and 26 persons, $15 \%$ of those who completed the PBM, scored above 4 , the cut-off point for burnout, which is higher compared with $7.1 \%$ in the Swedish national survey (Hallsten et al., 2002).

FSS Of the respondents, 176 persons (95\%) completed the FSS, and $63 \%$ of those scored 4 or above, whereas $43 \%$ scored above 5 . Mean score was 4.4 (SD =1.5), which means borderline fatigue, compared with 4.0 ( $\mathrm{SD}=$ 1.3) in a Norwegian normal sample of Lerdal et al. (2005). In a Canadian sample of persons with SCI this was 4.5 (SD =1.8) (FawkesKirby et al., 2008), and in Norwegian sample of polio-survivors this was $5.2(\mathrm{SD}=1.5)$ (Schanke \& Stanghelle, 2001).

Correlations between independent variables, mediators and dependent variables All tested variables demonstrated statistically significant correlations with each other. The strength of the relationship between the variables ranged from small (between coping and LTPA, $r=.268$ ) to large (the 
strongest relation was between PBS and Coping, $r=-.705)$. The correlation was negative between LTPA and the PDSS, the PBS and the FSS. The PEM correlated negatively with the same variables. Degree of coping correlated positively with LTPA, but negatively with the PDSS, the PBS and the FSS (see Table 2 for details).

\section{TABLE 2}

Mean scores and Pearson's correlations among variables

\begin{tabular}{|c|c|c|c|c|c|c|c|c|}
\hline Variables & Mean & $\mathrm{SD}$ & 1. & 2. & 3. & 4. & 5. & 6. \\
\hline 1. PDSS & 38.6 & 23.1 & - & & & & & \\
\hline 2. LTPA & 2.3 & .9 & $-.304 * *$ & - & & & & \\
\hline 3. PEM & 6.1 & 2.7 & $-.343 * *$ & $.516 * *$ & - & & & \\
\hline 4. Coping & 6.6 & 2.3 & $-.469 * *$ & $.268^{* *}$ & $.530 * *$ & - & & \\
\hline 5. PBS & 2.8 & 1.2 & $.564 * *$ & $-.319 * *$ & $-.489 * *$ & $-.705^{* *}$ & - & \\
\hline 6. FSS & 4.4 & 1.5 & $.431 * *$ & $-.312 * *$ & $-.464 * *$ & $-.554 * *$ & $.628 * *$ & - \\
\hline
\end{tabular}

**. Correlation is significant at the 0.01 level (2-tailed). Abbreviations: PDSS: physical disability stress scale; LTPA: Leisure time physical activity; PEM: Perceived exercise mastery; PBS: Pines burnout score; FSS: Fatigue severity scale

\section{The role of exercise in burnout and fatigue}

\section{FIGURE1}

Conceptual models used for the mediation analyses.

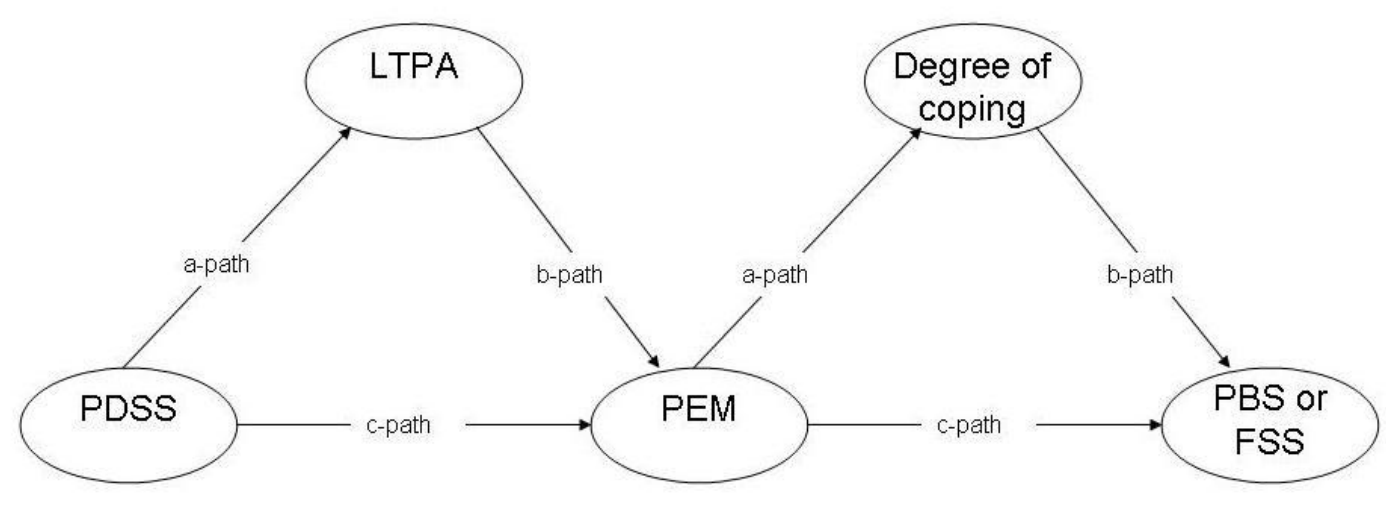

Abbreviations: PDSS: physical disability stress scale; LTPA: Leisure time physical activity; PEM: Perceived exercise mastery; PBS: Pines burnout score; FSS: fatigue severity scale. ${ }^{* *}$ : Significant at the 0.01 level (2-tailed).

Figure 1 illustrates the conceptual models used for the mediation analyses. First, we tested the relationship between PDSS and PEM. This relationship was mediated by LTPA (model 1). Then, we tested the relationship between the VAS measuring PEM and PBS, which was mediated by degree of coping (model 2).
Finally, we tested the relationship between the VAS measuring PEM and FSS, which was mediated by degree of coping (model 3 ). The criterion for mediation as suggested by Baron \& Kenny (1986) was used. In total, all the paths tested in the 3 models were significant; see Table 3 and Figure 1. The models were 
tested separately for mediation with three simple regression equations. In model 1 , the regression of the mediator (LTPA, path a) demonstrated a significant effect on the independent variable $(\mathrm{PDSS})(\mathrm{R} 2 \mathrm{adj}=.09, \mathrm{~F}=$ 17.614, $\mathrm{p}<$ 0.001). Second, the dependent variable (PEM, path c) significantly affected the independent variable (PDSS), $(\mathrm{R} 2 \mathrm{adj}=.11$, $\mathrm{F}=21.474, \mathrm{p}<0.001)$. Finally, the mediator significantly affected the dependent variable (PEM) in a regression model with both LTPA and PDSS as predictors. In this equation,
PDSS affected PEM less than in the third equation. See Table 3 for details. Model 2 and model 3 were tested in the same order, for details, see Table 3. The results of the mediation analyses demonstrated that PDSS was indirectly linked to PEM through LTPA $(\mathrm{R} 2 \mathrm{adj}=.27, \mathrm{~F}=31.839, \mathrm{p}<.001)$, that PEM was indirectly linked to burnout through degree of coping $(\mathrm{R} 2 \mathrm{adj}=.51, \mathrm{~F}=82.777, \mathrm{p}<$ .001 ), and lastly, that PEM was indirectly linked to FSS through degree of coping R2adj $=.36, \mathrm{~F}=45.808, \mathrm{p}<.001)$.

\section{TABLE 3}

Linear Models testing: PDSS $\rightarrow$ LTPA $\rightarrow$ PEM $\rightarrow$ Coping $\rightarrow$ PBS or FSS

\begin{tabular}{ccccc}
\hline \hline Variables and paths & $\Delta \mathrm{R} 2$ & $\beta$ & $\mathrm{t}$ & $\mathrm{p}$ \\
\hline Model 1: PDSS $\rightarrow$ LTPA $\rightarrow$ PEM & & & & \\
\hline LTPA as mediator of PEM & & & & \\
Path a, PDSS $\rightarrow$ LTPA & .09 & -.304 & -4.197 & .000 \\
Path b, LTPA $\rightarrow$ PEM & .26 & .516 & 7.775 & .000 \\
Path c, PDSS $\rightarrow$ PEM & .11 & -.343 & -4.634 & .000 \\
Path c + path b & .27 & & & \\
PDSS & & -.197 & -2.779 & .006 \\
LTPA & & .434 & 6.112 & .000 \\
\hline PTA & & & & \\
Model 2: PEM $\rightarrow$ Coping $\rightarrow$ PBS & & & & \\
Coping as mediator of PBS & & & & \\
Path a, PEM $\rightarrow$ Coping & .28 & .530 & 7.933 & .000 \\
Path b, Coping $\rightarrow$ PBS & .49 & -.705 & -12.705 & .000 \\
Path c, PEM $\rightarrow$ PBS & .23 & -.448 & -7.092 & .000 \\
Path c + path b & .51 & & & \\
PEM & & -.161 & -2.444 & .016 \\
Coping & & -.624 & -9.479 & .000 \\
\hline PEM & & & & \\
Podel 3: PEM $\rightarrow$ Coping $\rightarrow$ FSS & & & & \\
Path a, PEM $\rightarrow$ Coping & .28 & .530 & 7.933 & .000 \\
Path b, Coping $\rightarrow$ FSS & .30 & -.554 & -8.662 & .000 \\
Path c, PEM $\rightarrow$ FSS & .21 & -.464 & -6.704 & .000 \\
Path c + path b & .36 & & \\
PEM & & -.268 & -3.606 & .000 \\
Coping & & -.418 & -5.621 & .000 \\
\hline
\end{tabular}

Abbreviations: PDSS: physical disability stress scale; LTPA: Leisure time physical activity; PEM: Perceived exercise mastery; PBS: Pines burnout score; FSS: fatigue severity scale.

\section{DISCUSSION}

In this study we examined the prevalence of burnout and fatigue within a sample of persons living with incomplete SCI for more than two years. We observed a markedly higher prevalence of both burnout and fatigue compared to able-bodied populations (Hallsten et al., 2002; Krupp et al., 1989). In addition, we analysed the role of physical exercise in the 
association between stress caused by the disability and burnout or fatigue. We hypothesised that the PDSS was indirectly linked to PEM through LTPA, and that PEM was indirectly linked to burnout and fatigue through the individual's degree of coping with their everyday situation. All the findings in the mediator tests were consistent with this hypothesis.

In the literature, it is difficult to find information about the prevalence of burnout in the general population. In a Swedish national survey of the general adult population, the prevalence of burnout measured by the PBM was lower compared with the present study (7.1\% versus $15 \%$ respectively) even if the PBM mean score did not differ (Hallsten et al., 2002). The comparable mean score could partly be explained by the difference in sample sizes and that the distribution of the PBS in our sample was slightly skewed (range 5.7 and skewness 1.12 to the lower value). As observed, it is, however, likely that persons struggling in their everyday life to overcome their physical limitations in order to be as independent as possible, are more likely to experience a state of burnout than the general population. In addition, for persons with incomplete SCI, the functional improvements diminish over the years, and a decline in daily function may occur because of complications, possible overload, or normal aging. They will probably perceive a reduced sense of accomplishment, even if they exercise, which is considered a causal factor both in workrelated and athlete burnout (Lemyre et al. 2008).

The prevalence of the symptom of fatigue, as measured by the FSS in the present study, indicated that fatigue is much more common than burnout. This result is supported by other studies, both for persons with SCI and other neurological impairments (Anton et al., 2008; Fawkes-Kirby et al., 2008; Herlofson \& Larsen, 2002; Krupp et al., 1989; Schanke \& Stanghelle, 2001). For the general population, Lerdal et al. (2005) reported data from the FSS that revealed that scores were higher for persons with chronic illness (lasting 6 months or more). To avoid over-diagnosing high fatigue levels, they suggested that the threshold for high fatigue probably should be 5 on the FSS, instead of 4 , as had been suggested originally. In the present study, as many as $43 \%$ scored above 5 on the FSS, compared with $23 \%$ in the general population as reported by Lerdal et al (2005). In addition, $20 \%$ in the present study, scored between 4 and 5 on the FSS, indicating a borderline state of fatigue. It is argued that an overloading process is triggering the onset of both chronic fatigue syndrome (CFS) and burnout, and that fatigue is one of the symptoms in both concepts (Leone et al., 2010). The present findings emphasised how vulnerable persons with incomplete SCI are for both burnout and fatigue compared with the general population.

Furthermore, Leone et al. (2010) argued that CFS and burnout are different concepts, but that the relationship between them should be examined further. In a sport context, Lemyre et al. (2008) argued long-lasting physical exhaustion may contribute to the development of burnout. In this study, we can speculate whether fatigue, as a symptom, is a precursor of burnout, and that a person with a high level of fatigue may be at particularly risk of developing burnout. For persons with reduced physical capacity, like those with incomplete SCI, it is especially important to be aware of the consequences of neglecting the symptom of fatigue. Persons with incomplete SCI have a relatively unpredictable potential for recovery (Burns \& Ditunno, 2001). This may result in unrealistic expectations of their own physical capacity, and, consequently, they use less adaptive aids than needed in everyday life. Fawkes-Kirby et al. (2008) argued that lack of use of adaptive aids of persons with incomplete SCI was one of the reasons for fatigue. In addition, they registered that persons with incomplete SCI reported more severe fatigue than those with complete lesions. The results in the present study supported that those who experienced burnout, also experienced fatigue, and that the symptom of fatigue seemed to be a precursor for burnout.

Following the pathways in Figure 1, the mediating effects of both LTPA and the degree of coping seemed to buffer the negative effects due to stress. This is consistent with earlier research. Martin Ginis et al. (2003) demonstrated that changes in pain and stress improved well-being, and Latimer et al. (2005) found preliminary support for exercise in buffering the effects of stress on well-being.

Gerhart, Weitzenkamp, Kennedy, Glass and Charliefue (1999) reported various correlates of stress in long-term SCI from a longitudinal 
study, and Quale, Skanke, Froslie and Roise (2009) reported from a cohort study on posttraumatic stress symptoms in severely injured patients but neither of the studies found a relationship between stress and severity of disability. This finding is consistent with the present study where no differences in PDSS were found between the subgroups tetraplegia versus paraplegia.

In the present study, two pathways demonstrated an indirect association between stress and fatigue. First, PDSS and LTPA explained $27 \%$ of the variance in PEM, and secondly, PEM and the degree of coping explained as much as $37 \%$ of the variance in FSS. A stronger association was found between PEM and PBS where PEM and degree of coping explained $51 \%$ of the variance in PBS. This means that the associations between the variables in the study are stronger for PBS than for fatigue. This may be explained by the fact that the measure of burnout relies on psychological processes but the measure of fatigue is of a more physical nature.

In order to control for the reported amount of exercise, two different measures were used. A limitation in the use of IPAQ in this population was demonstrated. The IPAQ measures were highly skewed towards zero, and as a result did not capture the variation in the responses in the relative low physically active population. The response alternatives in LTPA included 2 responses for less active persons, either sedentary activities, or low intensity at least 4 hours per week. This seemed to be enough to statistically achieve a normal distribution, and thereby capture more of the variance in the low physical active population.

A limitation of this study may be the crosssectional design and use of self-reports. Also, social desirability may result in over-reporting of exercise (Sallis \& Saelens, 2000). Another limitation was the sample size, and that we do not know anything about the $37 \%$ that did not respond to the survey, which limits the generalisation. There is a possibility that persons with more complications or a more severe impairment, even if this is a very inactive population, are less likely to participate in a survey like this. The comments from the seven respondents who refused to participate indicated severe complications or functional limitations.

\section{Perspective}

This study examined the prevalence of burnout and fatigue within a sample of persons with incomplete SCI. In addition, we analysed the role of physical exercise and degree of coping in the association between stress caused by the disability and burnout or fatigue. This sample of persons with incomplete SCI perceived burnout and fatigue more frequently than the general population. Physical exercise and degree of coping buffered the associations between stress caused by the disability and both fatigue and burnout. Further research is needed. We need more knowledge about the physiological variations found among those with incomplete SCI. We also need to know more about the association between the physiological and psychological variables and how this association may influence degree of coping.

\section{Acknowledgements}

This project has been financially supported by the Birgit and Rolf Sunnaas Foundation. Special thanks to the respondents for sharing their experiences, and to Grete Sofie Anmarkrud, PT, Sunnaas Rehabilitation Hospital for her help in data-collection and data-computing. Thanks to Professor Marit Sørensen, Department of Coaching and Psychology, Norwegian School of Sport Sciences for her valuable comments and suggestions during manuscript preparation.

\section{REFERENCES}

Aires, N., Selmer, R., \& Thelle, D. (2003). The validity of self-reported leisure time physical activity, and its relationship to serum cholesterol, blood pressure and body mass index. A population based study of 332,182 men and women aged 40-42 years. European Journal of Epidemiology, 18, 479-485.

Aitken, R. C. (1969). Measurement of feelings using visual analogue scales. Procedings of the Royal Society of Medicine, 62, 989-993.

Anton, H. A., Miller, W. C., \& Townson, A. F. (2008). Measuring fatigue in persons with spinal cord injury. Archives of Physical Medicine and Rehabilitation, 89, 538-542.

Baron, R. M., \& Kenny, D. A. (1986). The moderator-mediator variable distinction in social psychological research: conceptual, 
strategic, and statistical considerations. Journal of Personality and Social Psychology, 51, 1173-1182.

Buchholz, A. C., McGillivray, C. F., \& Pencharz, P. B. (2003). Physical activity levels are low in free-living adults with chronic paraplegia. Obesity Research, 11, 563-570.

Burns, A. S., \& Ditunno, J. F. (2001). Establishing prognosis and maximizing functional outcomes after spinal cord injury: a review of current and future directions in rehabilitation management. Spine, 26, S137-S145.

Chaudhuri, A., \& Behan, P. O. (2004). Fatigue in neurological disorders. Lancet, 363, 978988.

Craig, C. L., Marshall, A. L., Sjostrom, M., Bauman, A. E., Booth, M. L., Ainsworth, B. E. et al. (2003). International physical activity questionnaire: 12 -country reliability and validity. Medicine and Science in Sports and Exercise, 35, 1381-1395.

Eriksen, H. R., Murison, R., Pensgaard, A. M., \& Ursin, H. (2005). Cognitive activation theory of stress (CATS): from fish brains to the Olympics. Psychoneuroendocrinology, 30, 933-938.

Fawkes-Kirby, T. M., Wheeler, M. A., Anton, H. A., Miller, W. C., Townson, A. F., \& Weeks, C. A. (2008). Clinical correlates of fatigue in spinal cord injury. Spinal Cord, 46, 21-25.

Furlong, M., \& Connor, J. P. (2007). The measurement of disability-related stress in wheelchair users. Archives of Physical Medicine and Rehabilitation, 88, 12601267.

Gerhart, K. A., Weitzenkamp, D. A., Kennedy, P., Glass, C. A., \& Charlifue, S. W. (1999). Correlates of stress in long-term spinal cord injury. Spinal Cord, 37, 183-190.

Graff-Iversen, S., Anderssen, S. A., Holme, I. M., Jenum, A. K., \& Raastad, T. (2008). Two short questionnaires on leisure-time physical activity compared with serum lipids, anthropometric measurements and aerobic power in a suburban population from Oslo, Norway. European Journal of Epidemiology, 23, 167-174.

Hallsten, L., Bellagh, K., \& Gustafsson, K. (2002). Burnout in Sweden: a national survey. (Rep. No. 6). Stockholm, Sweden: Arbetslivsinstitutet.
Harvey, L. A., Lin, C. W., Glinsky, J. V., \& De, W. A. (2009). The effectiveness of physical interventions for people with spinal cord injuries: a systematic review. Spinal Cord, 47, 184-195.

Herlofson, K., \& Larsen, J. P. (2002). Measuring fatigue in patients with Parkinson's disease - the Fatigue Severity Scale. European Journal of Neurology, 9, 595-600.

Kennedy, P., Evans, M. J., Berry, C., \& Mullin, J. (2003). Comparative analysis of goal achievement during rehabilitation for older and younger adults with spinal cord injury. Spinal Cord, 41, 44-52.

Krupp, L. B., LaRocca, N. G., Muir-Nash, J., \& Steinberg, A. D. (1989). The fatigue severity scale. Application to patients with multiple sclerosis and systemic lupus erythematosus. Archives of Neurology, 46, 1121-1123.

Lannem, A. M., \& Sorensen, M. (in review). The role of physical exercise, perceived exercise mastery and stress in persons with incomplete Spinal Cord Injury (SCI).

Lannem, A. M., Sorensen, M., Lidal, I. B., \& Hjeltnes, N. (2010). Perceptions of exercise mastery in persons with complete and incomplete spinal cord injury. Spinal Cord, 48, 388-392.

Latimer, A. E., Martin Ginis, K. A., \& Hicks, A. L. (2005). Buffering the effects of stress on well-being among individuals with spinal cord injury. Therapeutic Recreation Journal, 39, 131-138.

Lemyre, P. N., Hall, H. K., \& Roberts, G. C. (2008). A social cognitive approach to burnout in elite athletes. Scandinavian Journal of Medicine and Science in Sports, 18, 221-234.

Leone, S. S., Wessely, S., Huibers, M. J., Knottnerus, J. A., \& Kant, I. (2010). Two sides of the same coin? On the history and phenomenology of chronic fatigue and burnout. Psychology and Health, 1-16.

Lerdal, A., Wahl, A., Rustoen, T., Hanestad, B. R., \& Moum, T. (2005). Fatigue in the general population: a translation and test of the psychometric properties of the Norwegian version of the fatigue severity scale. Scandinavian Journal of Public Health, 33, 123-130.

Marino, R. J., Barros, T., Biering-Sorensen, F., Burns, S. P., Donovan, W. H., Graves, D. E. et al. (2003). International standards for 
neurological classification of spinal cord injury. J Spinal Cord Med, 26 Suppl 1, S50S56.

Martin Ginis, K. A., Latimer, A. E., McKechnie, K., Ditor, D. S., McCartney, N., Hicks, A. L. et al. (2003). Using exercise to enhance subjective well-being among people with spinal cord injury: the mediating influences of stress and pain. Rehabilitation Psychology, 48, 157-164.

Maslach, C. (1993). Burnout. A multidimensional perspective. In W.B.Schaufeli, C. Maslach, \& T. Marek (Eds.), Professional Burnout. Recent Developments in Theory and Research (pp. 19-32). Washington, DC: Taylor \& Francis.

Pines, A., \& Aronson, E. (1988). How Burned Out Are You? In Career Burnout: Causes and Cures. (2. ed., pp. 218-222). New York: The Free Press, A divition of Macmillan, Inc.

Quale, A. J., Schanke, A. K., Froslie, K. F., \& Roise, O. (2009). Severity of injury does not have any impact on posttraumatic stress symptoms in severely injured patients. Injury, 40, 498-505.

Raedeke, T. D. (1997). Is athlete burnout more than just stress? A Sport Commitment Perspective. Journal of Sports and Exercise Psychology, 19, 396-417.

Raedeke, T. D., \& Smith, A. L. (2001). Development and preliminary validation of an athlete burnout measure. Journal of Sports and Exercise Psychology, 19, 396417.

Roberts, G. C., \& Kristiansen, E. (2012). Goal setting to enhance motivation in sport. In G. C. Roberts \& D. C. Treasure (Eds.), Advances in motivation in sport and exercise (3rd ed.). Champaign, IL: Human Kinetics.

Saebu, M., \& Sorensen, M. (2010). Factors associated with physical activity among young adults with a disability. Scandinavian Journal of Medicine and Science in Sports.

Sallis, J. F., \& Saelens, B. E. (2000). Assessment of physical activity by selfreport: status, limitations, and future directions. Res.Q.Exerc.Sport, 71, S1-14.

Saltin, B., \& Grimby, G. (1968). Physiological analysis of middle-aged and old former athletes. Comparison with still active athletes of the same ages. Circulation, 38, 1104-1115.
Schanke, A. K., \& Stanghelle, J. K. (2001). Fatigue in polio survivors. Spinal Cord, 39, 243-251.

Schaufeli, W. B., Enzmann, D., \& Girault, N. (1993). Measurement of burnout. In Schaufeli W.B., Maslach, C., \& Marek, T. (Eds.), Professional Burnout: Recent Developments in Theory and Research. (pp. 199-215). Philadelphia: Taylor \& Francis.

Sorensen, M. (1997). Self-referent thoughts in exercise: The self-perception in exercise questionnaire. European Journal of Phsychological Assessment, 13, 195-205.

Ursin, H., \& Eriksen, H. R. (2004). The cognitive activation theory of stress. Psychoneuroendocrinology, 29, 567-592.

Wessely, S. (2001). Chronic fatigue: symptom and syndrome. Annals of Internal Medicine, 134, 838-843.

Wilhelmsen, L., Tibblin, G., Aurell, M., Bjure, J., Ekström-Jodal, B., \& Grimby, G. (1976). Physical activity, physical fitness and risk of myocardial infarction. Advances in Cardiology, 18, 217-230.

Wilhelmsen, M. (2009). Samordnet levekårsundersøkelse 2008.Tverrsnitts undersøkelsen. (Coordinated investigation of living conditions) (Rep. No. 2009/40). Statistics Norway.

\section{Corresponding author:}

Anne M. Lannem

E-mail address: a.m.lannem@nih.no 